\title{
OAMIAN \\ AIAA 2000-2016 \\ Controlling Chaos Via Knowledge of Initial Condition for a Curved Structure
}

L. Maestrello

NASA Langley Research Center

Hampton, VA 23681-2199

6th AIAA/CEAS Aeroacoustics Conference 12-14 June 2000
Lahaina, Hawaii

For permission to copy or republish, contact the American Institute of Aeronautics and Astronautics, 1801 Alexander Bell Drive, Sulte 500, Reston, Virginia 20191-4344. 
$A I A \Lambda-2000-2016$

\title{
CONTROLLING CHAOS VIA KNOWLEDGE OF INITIAL CONDITION FOR A CURVED STRUCTURE
}

\author{
Lucio Maestrello \\ NASA Langley Research Center. \\ Hampton, VA 23681-2199
}

\begin{abstract}
Nonlinear response of a flexible curved panel exhibiting bifurcation to fully developed chaos is demonstrated along with the sensitivity to small perturbation from the initial conditions. The response is determined from the measured time series at two fixed points. The panel is forced by an external nonharmonic multifrequency and monofrequency sound field. Using a low power time-continuous feedback control, carefully tuned at each initial condition, produces large long-term effects on the dynamics toward taming chaos. Without the knowledge of the initial conditions, control may be achieved by destructive interference. In this case, the control power is proportional to the loading power. Calculation of the correlation dimension and the estimation of positive Lyapunov exponents, in practice, are the proof of chaotic response.
\end{abstract}

\section{Backround}

Dynamical instability and chaos are now established to be common features of many nonlinear processes in engineering, physics, climatology, medicine, astronomy, biology, and ecology. Chaos manifests beneficial as well as destructive effects. Detecting nonlinearity is considerably easier than identifying chaotic dynamics. Positive identification of chaos is obtained by estimating the correlation dimension and the Lyapunov exponent from an experimental time series. Structural dynamics and acoustics responses have demonstrated the existence of strong nonlinear behaviors when forced by high acoustic loading; as a result, appropriate dynamical control techniques have been implemented to stabilize the system. The control algorithm proposed by Ott. Grebogi, and Yorke' has been used in many applications to stabilize an unstable periodic orbit through the application of small, carefully selected perturbations aimed at establishing control over chaos response. ${ }^{2-8}$
The present experiment is designed to control the chaotic response via initial conditions by changing the system dynamics on a curved panel structure loaded by high intensity sound. Instability and chaos are two of the main concepts associated with nonlinear vibration, which have revolutionized our understanding about the response of a dynamical system. Because there is no universal set of eigen functions for a nonlinear system, most data analyses are performed in time domain. Deterministic data have complicated dynamics, and prediction is impossible over extended time; however, we do the experiments to gain insight into our limitations set by nonlinearly. ${ }^{9-11}$ In our experiments, the linear system fails to describe the structure response forced by high intensity sound. Stochastic measurements are not useful because they concentrate on their average such as correlation coefficient and autocorrelation function, and they are not able to distinguish between the data from the linear system and those from the nonlinear system, whereas short term reveals the time limit of a reliable prediction. One cannot repeat the experiment exactly because of the changes in the initial condition, since it is one of the most peculiar features of a nonlinear chaotic response, Abarbanel. ${ }^{12}$ In a previous experiment, a panel forced by constant or accelerated flow and sound exhibits different dynamics; this indicates the dependency on the unpredictable initial condition in each run. ${ }^{8}, 13$ Furthermore, tension and curvature of the structure depend on the loading, and they constitute a coupling with the response; one manifestation is the spontaneous surface deformation. Some related problems are elastic structure forced by jet noise, turbulent boundary layer at constant and accelerated speed, and flutter behaviors such as wing and panel with various nonlinearities. ${ }^{14-19}$ Another example related to medical science is that the heart under normal conditions has a periodic rhythm; however, as one nears a heart attack, it becomes chaotic. ${ }^{20}$ Experimentally we explore how chaotic response of a flexible curved panel forced by an 
external single and nonharmonic multifirequency sound can be controlled by using active control with the knowledge of the initial condition. Without the knowledge of the initial condition, control is achievable by the equilibrium of forces and by damping. ${ }^{21}$ Traditional control methods have avoided chaotic responses, which comprise a large and rich part of the physical system.

The initial condition is unknown in a time series of unknown origin. Therefore a technique is introduced to determine the initial condition by using the time series of the experimental data. $10,22,23$ From the data, we want to determine whether the system response is chaotic by using techniques drawn from classical dynamics. One can argue that the time series has been generated by random field; however, the definition of chaos includes three elements: (a) determinism, (b) aperiodicity, and (c) sensitive dependence on initial conditions, Ruelle 24 and Abarbanel. ${ }^{25}$ This paper begins by describing the instrumentation followed by the description of a technique used to determine the initial condition and by the description of the panel response and the method used for active control. The paper is organized as follows: Section 2 discusses instrumentation. Analysis of the data is reported in section 3. Results are given in section 4, with section 4.1 describing the wall pressure, section 4.2 describing the panel response, section 4.2.1 describing a search of initial condition, section 4.2 .2 describing panel response and active control from multifrequency sound, and section 4.2 .3 describing panel response and active control from monofrequency sound. Section 5 discusses the correlation dimensions and Lyapunov exponents.

\section{Instrumentation}

The experiment is set up to study the nonlinear response of panels excited by plane acoustic waves at normal and oblique incidences. The panels are curved airplane fuselage types made of aluminum machined from a plate into two panels separated by a longitudinal tear stopper with a $0.5-\mathrm{cm}$ radius of curvature to minimize the amplitude of the reflected and transmitted waves. The structure is $0.609 \mathrm{~m}$ wide, $1.019 \mathrm{~m}$ long ( $1 / 3$ of the actual length), and $0.0109 \mathrm{~cm}$ thick, with a radius of curvature of 2.529 $\mathrm{m}$. The panels are mounted in a rigid absorbing partition dividing two anechoic rooms, the source and the transmission rooms. (See Figure 1.) The acoustic sources are crealted by four 120-Walt phatseamplitude matched speakers mounted on a ditfuser facing the structure with $138 \mathrm{~dB}$ acoustic power at $687 \mathrm{~Hz}$. The wall pressure tluctuations are measured with miniature pressure transducers mounted tlush within the tear stopper between panels. The vibration response is measured with two miniature accelerometers located at one-quarter length and three-quarter length of the panel on the centerline. The transmitted pressure is measured with a pressure transducer. All measurements are made from directcurrent response.

\section{Experimental Technique and Data Analysis}

The nonlinear time series for short-time signal processing is the basic tool used to analyze the experimental data. They are applied to input and transmitted pressure and panel response. A limited number of dynamic variables and positions can be measured on the panel. The key element in resolving the problem is that the full system phase space has one-to-one correspondence with the measurements of a limited number of point and variables. Local properties of the dynamics are as follows:

1. Energy variance is a technique used to locate unstable orbit and consists of timeseries, spectrum, phase portrait, probability, and Poincaré map

2. Initial condition determines whether the trajectory of the attractor diverges exponentially

3. Correlation dimension is the number of degrees of freedom

4. Lyapunov exponent gives the statistical quantities that indicate uncertainty

The time history of the wall pressure fluctuation and panel acceleration is measured, and the power spectral density, the phase portrait, and the probability distribution are computed from it. For a nonstationary signal $q(t, x)$, such as the pressure fluctuation $p(t, x)$ of the panel acceleration $g(t, r)$, the instantaneous power spectrum at instant $T$ is defined by

$$
P(f, T)=\left|\frac{1}{2 \pi} \int_{T-1 / 2}^{T+1 / 2} \exp (i 2 \pi f t) q(t, x) d t\right|^{2}
$$

where $T$ is chosen so that the experimental run contains the interval $T-l / 2, T+/ / 2$ for a sufficiently 
large 1 . The probibility density of $q(T, x)$ is denoted by $Q(r, T)$, where

$$
\begin{gathered}
\hat{q}(T, x)=\frac{1}{l} \int_{T-1 / 2}^{T+1 / 2} q(t, x) d t \\
Q(r, T)=\frac{d}{d r} \operatorname{prob}[\hat{q}(T, x) \leq r]
\end{gathered}
$$

In chaotic dynamics, searching for a lowdimensional characterization of the system is of great interest. Let $q(t, x)$ be a measured temporal signal or time series at position $x$, which is embedded in a $d$-dimensional phase space by a time delay $\tau$. The set $Z(t)=\left[z_{1}(t), \ldots, z_{d}(t)\right]$ is regarded as a trajectory in the $d$-dimensional phase space. The distance between two points $Z\left(t_{i}\right)$ and $Z\left(t_{j}\right)$ is given by $d_{i j}$, and for a small $\varepsilon>0$, let $N_{d}(n, \varepsilon)$ be the number of pairs of points with distance $d_{i j}<\varepsilon$. Then the correlation sum $C_{d}(\varepsilon)$ and the correlation dimension $D(d)$, for given $d$, are defined by Grassberger and Procaccia ${ }^{26}$ as

$$
\begin{gathered}
C_{d}(\varepsilon)=\lim _{n \rightarrow \infty} \frac{2 N_{d}(n, \varepsilon)}{n(n-1)} \\
D_{d}=\lim _{\varepsilon \rightarrow 0} \frac{\log C_{d}(\varepsilon)}{\log \varepsilon}
\end{gathered}
$$

For computation, the parameters $\tau$ and $d$ must be chosen properly, and the correlation dimension $D_{d}$ is estimated by

$$
D_{d}=\frac{\log C_{d}(n, \varepsilon)}{\log \varepsilon}
$$

for a sufficiently small $\varepsilon$ and large $n$. The estimated dimension $D$ is taken as the asymptotic value of $D_{d}$ as the embedding dimension $d$ increases.

Given the estimated dimension $D$ and using the method advocated by Grassberger and Procaccia, ${ }^{26}$ the Lyapunov exponent, which is one of the most important characteristics in the dynamics system, can be approximately computed. Several methods exist for computing the Lyapunov exponents. ${ }^{12}, 22,24$, 26-29 The Eckmann-Ruelle method ${ }^{28}$ is used herein. Consider $Z(t)$ as the trajectory of a dynamic system in the phase space of dimension $d=D$, obtain the tangent (linear) maps $T_{i}=1,2, \ldots, k$ of this reconstructed dynamical system by a least-squares fit, decompose $T_{i}$ into an orthogonal matrix $Q_{i}$ and an upper triangular matrix by $T_{1}-Q_{1} R_{1}$ and $T_{i} Q_{i-1}=Q_{1} R_{i}$, for $i \geq 2$. and compute the Lyapunov exponents as

$$
\lambda_{i}=\lim \frac{1}{(k-1)} \log \left|\left(R_{k-1} \ldots R_{2} R_{1}\right)_{i i}\right|
$$

for $i=1,2, \ldots, D$. For details see Eckmann-Ruelle algorithm given by Conte and Dubois ${ }^{27}$ and Maestrello. ${ }^{13}$

\section{Results}

Time series for short-time signal processing is applied to identify the input wall pressure and panel response to stabilize a complex surface vibration. The time series of chaotic signals has broadband components, and we select conditions such that the data are of low dimension where the noise is of minimum influence.

\subsection{Wall Pressure}

Now look at the input acoustic field. Some details of the analysis of the experimental results along with physical argument are given. The surface pressure fluctuations with $138 \mathrm{~dB}$ input acoustic power were measured on the rigid surface of the tear stopper between panels because the pressure transducer cannot be mounted on the vibrating panel without altering the response (Figure 1). The loading is induced by the sound from four nonharmonic frequencies corresponding to $f_{1}=387, f_{2}=425$, $f_{3}=512$, and $f_{4}=687 \mathrm{~Hz}$. The measured real-time pressure $p(t)$ of the time series over the interval $T$, the computed power spectral density $P(f, T)$, the phase plots of the computed $\dot{p}(t)$ versus $p(t)$, and the computed probability density $Q(r, T)$ are shown in Figure 2. The real-time wall pressure $p(t)$ shown for an interval of $0.04 \mathrm{sec}$ near the instant $T$ is used to evaluate the spectrum, phase, and probability. The time series of the data is not periodic over short or over an extended time, the spectrum is dominated by four basic forcing frequencies and harmonics, the phase plot indicates divergency and nonsymmetry in time, and the probability plot is clearly non-Gaussian with large standard deviation. Simultaneous spatial pressure measurements along the tear stopper indicate minor changes in amplitude and phase. The analysis suggests that the wall pressure data came from few degrees of freedom, and it is natural to look to the low frequencies and large scale as the origin of the low-dimensional signals. 


\subsection{Pind Responsic}

Charotic time series are observed routinely in our experiments. The characterization of irregular. broadband response is generic in nonlinear dynamic, and the extraction of physically and useful information including controlling uncorrected region of chaos is part of the following presentation.

\subsubsection{Search for Initial Forcing Parameters on}

Long-Term Response. It is well known that the periodic forcing of a nonlinear dissipative dynamical system can lead to chaos. A simple example is the Duffin-Holmes oscillator, ${ }^{30}$ a periodically forced Duffin equation. Similar to the sensitive dependence on the initial condition, the chaotic transition depends sensitively on the initial forcing condition, such as the amplitude and frequency of the forcing function. In fact it is such a sensitive dependence on the initial forcing parameter that has suggested the idea of using a weak periodic external force to control the chaos. In the case of Duffin-Holmes and related equations, such a control technique was illustrated in the papers by Braiman and Goldhirsch ${ }^{31}$ and Chacon and Bejarano ${ }^{32}$ among others.

In the experimental investigation, the dynamical system is governed by a nonlinear partial differential equation for curved elastic panel, which is difficult to deal with. As a crude simple model, regarding the panel as a lumped-parameter system is assumed to satisfy a generalized Duffin-Holmes equation such as

$$
\rho \frac{d^{2} w}{d t^{2}}+\alpha w+\beta w^{3}=p_{w}(a, \omega, t)
$$

where $\rho$ and $\alpha$ and $\beta$ are effective system parameters corresponding, respectively, to the density and the elastic constant, and $p$ is the initial forcing field of the wall pressure. In contrast with the periodically forced case, the forcing function is assumed to be quasiperiodic of the form:

$$
p(a, \omega, \iota)=\sum_{i=1}^{n} a_{i} \cos \left(\omega_{i^{t}}+\phi_{i}\right)
$$

with given forcing parameters $=$ the amplitudes $\left(a_{1}, a_{2}, \ldots, a_{n}\right)=a$, the circular frequencies $\left(\omega_{1}, \omega_{2}, \ldots, \omega_{n}\right)=\omega$, and the phases $\phi_{1}, \phi_{2}, \ldots, \phi_{n}$ where the frequencies are assumed to be incommensurate. Theoretically the transition to chaos caused by quasiperiodic forcing is not well understood. Based on the result of the monofrequency case mentioned previously. it is possible to suppose that the chaotic transition due to a quasiperiodic forcing depends on the initial forcing parameters $a_{i}$ and $\omega_{i}$ as well. The experimental result seems to confirm this conjecture.

In the experimental study, for the initial forcing field, the wall pressure $p$ is induced by an acoustic incident plane wave with four given frequencies: $f_{1}=387, f_{2}=425, f_{3}=512$, and $f_{4}=687 \mathrm{~Hz}$, where $f_{i}=\frac{\omega_{i}}{2 \pi}, i=1,2,3,4$.

To begin, the initial forcing amplitudes $a_{i}$ are kept small. As time progresses, the amplitude increases gradually to drive the system into chaotic state. In each run, the panel response $g(t)$ (the panel acceleration) and transmitted acoustic pressure $p_{r}(t)$ are measured. The results show that the chaotic panel response depends strongly on the forcing conditions, the parameters $a_{i}$ in this case. The acceleration response $g(t)=\frac{d^{2} w}{d t^{2}}$ of the panel, as the amplitudes $a_{i}$ increase in time to a set of constant postchaotic transition values, is shown in Figure 3 where a nonsymmetrical panel response $g(t)$ is indicated in the chaotic regime. Based on the measurement data, the finite time $T$, the wall pressure $p(t)$, the panel response $g(t)$, and the transmitted pressure $p_{r}(t)$ and then, by the Fourier transform, the corresponding spectral density functions of the wall pressure $P(f, T)$, the panel response $G(f, T)$, and the transmitted pressure $P_{r}(f, T)$ are calculated. At low frequency, a comparison of the spectral density is made in Figure 4. As expected, the wall pressure spectrum $P(f, T)$ shows peaks at the four initial frequencies $f_{1}$ to $f_{4}$. The panel response spectrum $G(f, T)$ exhibits robust peaks at the same four forcing frequencies, $f_{1}$ to $f_{4}$, and, in addition, at the subharmonics $1 / 2 f_{1}$ and $1 / 2 f_{2}$ as well as the harmonics $2 f_{1}$ and $2 f_{2}$. The other peak frequencies are incommensurable. The transmitted pressure spectrum $P_{r}(f, T)$ also shows the same four peaked forcing frequencies with subharmonics and harmonics. Thus, from the experimental evidence it is seen that at low frequencies, the four basic forcing frequencies $f_{1}$ to $f_{4}$ appear prominently in both the panel response and the transmitted pressure power spectra. 
To suppress the chass, in view of the sensitive dependence on the initial forcing parameters, it is sensible to use a weak quasiperiodic forcing $u(\varepsilon, \omega, \theta, \iota)$ as a control. In particular $u$ is taken to be

$$
u(\varepsilon, \omega, \theta, t)=\sum_{i=1}^{4} \varepsilon_{i} \cos \left(\omega_{i} t+\theta_{i}\right)
$$

where $\omega_{i}, i=1,2,3,4$, represents the initial forcing frequencies, and the amplitudes $\varepsilon_{1}, \varepsilon_{2}, \varepsilon_{3}$, and $\varepsilon_{4}$, which are small, and the phases $\theta_{1}, \theta_{2}, \theta_{3}$, and $\theta_{4}$ are to be adjusted experimentally to suppress the chaos. In practice the forcing frequencies $f_{i}$ may not be measured. Then, at least for the low-frequency case, they may be identifiable, amid the subharmonics and harmonics, from the peak frequencies in the panel response and transmitted pressure power spectra $G(f, T)$ and $P_{r}(f, T)$. Such peak frequencies are selected as possible forcing frequencies as a trial control function $u$. The possibility is tested in sections 4.2 .2 and 4.2 .3 as part of the chaos control strategy.

4.2.2. Uncontrolled and Controlled Response From Multifrequency Loading. Measurements of the panel response are made simultaneously at two points along the centerline, at one-quarter and threequarters length. Figures 5 and 6 show the acceleration response $g(t)$ of the time series over the time interval $T$, the computed power spectral density $G(f, T)$, phase plot of the computed $\dot{g}(t)$ versus $g(t)$, and the computed probability density $\bar{Q}(r, T)$. Also, panel response $g(t)$ versus controller response $c(t)$ and controller response $c(t)$ are shown in Figure 5. A particular feature of the power spectra is the appearance of numerous incommensurate frequencies mixed with harmonics and subharmonics. The abrupt changes occurring in the time series have been previously observed ${ }^{33}$ to be the result of frequency locking among previously incommensurate frequencies. Then the appearance of chaos, behind the locking state, was described to be the loss of synchronization between frequency locked modes. The temporal evolution of the power spectra showed pulsating chaotic behaviors from nonlinear coupling among modes waves. We determine whether the nonlinear determinism can be detected even when mixed with probable noise from the measured time series. We estimate the dimension and Lyapunov exponent over a finite time interval $T$. The results indicate that a signiticant number of exponents are positive. (See section 5.) The response of the two accelerometers symmetricially placed on the panel indicates loss of correlation for high input and indicate correlation at lower input level. The difterence in response between accelerometers is shown by the time series in Figures $5(a)$ and 6(a). and by the power spectra levels and distributions in Figures 5(b) and 6(b). The phase portrait plots are skewed indicating unsymmetrical response, and the probability plots are non-Gaussian with a larger standard deviation than at a lower level in Figures $5(\mathrm{c}), 5(\mathrm{~d}), 6(\mathrm{c})$, and $6(\mathrm{~d})$. The controller is virtually driven by the panel response in an uncontrolled state in Figures 5(e) and 5(f). In general, power spectra are very good for the visualization of periodic and quasiperiodic phenomena and their separation from chaotic time evolution. However, the analysis of chaotic responses themselves do not benefit much from the power spectra, because they lose phase information, which is essential for the understanding of what happens on the strange attractor. In the latter case, the dimension of the attractor is no longer related to the number of independent frequencies in the power spectrum, and the dimension has been related to the concept developed for the experimental technique and data analysis in section 3 , with details obtainable. 28,29

Active control of the panel response is achieved at low input power by using time-continuous feedback, carefully tuned at each initial condition frequency. 1 Active control is sensitively dependent on the initial condition; the knowledge of it is a requirement for achieving control via stability principles (section 4.2.1). The broadband response is generated by four frequencies: $f_{1}=387, f_{2}=425$, $f_{3}=512$, and $f_{4}=687 \mathrm{~Hz}$ (Figures $5(\mathrm{~b}$ ) and $6(\mathrm{~b})$ ). The controller is symmetrically placed between two accelerometers and tuned at all initial conditions. The amplitude increases at each forcing frequency when control is applied; the phases are adjustable for achieving control. Amplitude reduction starts at the highest frequencies. The amount is related to the power input to the controller for each initial condition frequency selected during the initial part of the run. As the amplitude of the forcing frequencies increase, reciprocally the amplitude of all other frequencies decreases with slope greater than $1 / f$. Results indicate that part of the energy is transferred from high to lower frequencies and part is dissipated. A different control mechanism was used in an earlier experiment in which the energy from the hightrequency harmonics, superimposed on a turbulent boundary layer in accelerated flow, accumulate into 
the fundamental during the control process with very low dissipattion. ${ }^{8}$ In our present experiment. control is achieved by actively forcing all four fundamentals (section 4.2.1) through phase and amplitude tuning procedures with several stage adjustments. In stages, the controller forces the panel with amplitude and phase variations so that the energy of the highfrequency band is shifted loward the forcing frequency. Active control is not straightiorward, and the desired control is not always achievable. The final results indicate that the medium-and highfrequency broadband response is reduced on the average by about 3 decays in amplitude. The time trace amplitude is also reduced, and the response changes into quasiperiodic state. The effectiveness of the active control is noticeable by the phase portrait and probability distributions. Further reduction may be obtainable by increasing the input control power; however, it may not be practical because the controller power approaches the loading power. Control is then achieved by destructive interference.

\subsubsection{Uncontrolled and Controlled Response} From Monofrequency Loading. To widen the scope of the experiments, monofrequency loading $f=1050$ $\mathrm{Hz}$ was used to illustrate the response and active control. Figures $7(a)$ and $7(b)$ show the uncontrolled and controlled power spectral density, an example in which the controller input is obtained with knowledge of the initial condition of the panel dynamics. Results indicate that monofrequency loading can induce broadband response. Active control reduces the broadband spectrum to a periodic spectrum, fundamental and harmonics. The harmonic frequencies maintain nearly constant amplitude, whereas the control spectrum from multifrequencies forces rapidly decay previously discussed (Figures 5 and 6).

Figure 7(c) shows an additional example of controlled power spectral density, in which the controller frequency $f=2100 \mathrm{~Hz}$ does not correspond to the forcing frequency $f=1050 \mathrm{~Hz}$. This is a case in which control is applied indiscreetly at the same (arbitrary) peak frequency. This example demonstrates when the control frequency does not correspond to the forcing frequency, control is not achievable via stability approach. Figure $7(\mathrm{c})$ indicates that spectrum level and distribution are virtually unaffected when compared with Figure $7(\mathfrak{a})$. From the experiment we learn that, when the control trequency does not correspond to the initial condition, frequency control is achievable by destructive interference when the control power approaches the loading power. On the other hand, when control is obtained with the use of the initial condition, the power required is very small.

\section{Correlation Dimension and Lyapunov Exponents}

The possibility of derived relationships between the correlation dimension $D$ and Lyapunov exponents by using the Grassberger and Procaccia algorithm has been previously described. ${ }^{26}$ The fundamental role played by Lyapunov exponents in defining chaotic dynamics has stimulated the search for a statistical framework within which the accuracy of estimated exponents might be quantified. ${ }^{24}$ Confidence bounds on estimation are of great interest. Experimental observations are limited, and it is in the finite time that Lyapunov exponents are of interest in our data analysis. First the dimension of the attractor was determined to be related to the number of degrees of freedom of the panel response, a method described by Ruelle; ${ }^{24}$ Screenivasan; ${ }^{34}$ Abraham, Gollub, and Swinney; ${ }^{9}$ and Sahay and Screenivasan. ${ }^{35}$ To compute the dimension, we choose a range of size over which the scaling is to be estimated. The number of dimensions of the panel response increases with the increase in acoustic power level. The computation procedures have been previously illustrated by Conte and Dubois ${ }^{27}$ and Maestrello. ${ }^{13}$

Figure 8 shows the computed exponents $\lambda_{2}, \lambda_{4}, \lambda_{6}$, versus the embedding time $(D-1) \tau$ obtained from the acceleration response of the panel from the time data typical of uncontrolled response in Figure 5(a). Only the values of the largest positive exponents are shown; most of the exponents are positive. Even a single positive exponent is sufficient proof that the attractor of the system is chaotic. As a conservative rule, 16 bits of precision are used for the experimental calculations. When the panel input acoustic loading is reduced, the largest Lyapunov exponent values decrease, and they become negative.

\section{Conclusions}

The active control of broadband chaos response on a panel structure with two selected examples has been illustrated. Many others could be cited. Much effort has been spent on the control of chaos, because in practice it may be impossible to avoid. We have observed the inherent sensitivity of the panel response to initial condition displaying chaotic behaviors. Chaos response was anticipated from the 
experimental time series and by the numbers of subharmonics and harmonics in the spectra. The chaotic state is of low dimension established by the Lyapunov exponent test. The loading is temporally chaotic and weakly spatiotemporally correlated.

We experimented with the controlling mechanism using small perturbations about the initial condition with controlling signal power far below the power produced by the chaotic system response. The complexity of chaos and the sensitivity to small perturbations to initial condition are combined to control the responses. We demonstrated stabilization and control of chaos on time-continuous feedback, carefully tuned with each initial condition. The feedback controller is easy to implement; it stabilizes broadband chaos on a panel efficiently and is resistive to exterior noise.

Destructive interference and damping are the two most popular control methods in the literature; however, they are not part of the rapid progress made in understanding the dynamics of deterministic nonlinear systems, especially chaos, dimensionality, Lyapunov exponents, initial condition, and control by low power feedback.

Finally, some practical guidelines indicate that active control is achievable because the initial conditions are determined. In a flight experiment where the structure response is related to the load, that is, the wall pressure difference, the initial condition needs to be determined in order that the stability control law may be effectively applied. These developments have raised further new interesting questions and potentialities.

\section{References}

1. Ott, E., Grebogi, C., and Yorke, J. A., "Controlling Chaos," Phys. Rev. Lett., Vol. 64, pp. 1196-1199, 1990.

2. Epureanu, B. I., and Dowell, E. H., "System Identification for the Ott-Grebogi-Yorke Controller Design," Phys. Rev. E, Vol. 56, No. 5. pp. 5327-5331, 1997.

3. Ditto, W. L., Rauseo, S. N., and Spano, M. L., "Experimental Control of Chaos," Phys. Rev. Letr., Vol. 65, pp. 3211-3214, 1990.

4. Hunt, E. R., "Stabilizing High-Periodic Orbits in a Chaotic System; The Diode Resonator," Phys. Lett., Vol. 67, p. 1953, 1991.
5. Petruv, V., Peng. B. O., and Shuwalter, K., "A Map-Based Algorithm for Controlling Low Dimensional Chaos," J. Chem. Phys., Vol. 96. pp. 7506-7513, May 1992.

6. Gartinkel, A., Spano, M. L., Ditto, W. L., and Weiss, J., "Controlling Cardiac Chaos," Science, Vol. 257, pp. 1230-1235, 1992

7. Chow, P. L., and Maestrello, L., "Vibration Control of a Nonlinear Elastic Panel," ICASE Rep. 98-46, NASA/CR-1998-208734, 1998.

8. Maestrello, L., "Active Control of Panel Oscillation Induced by Accelerating Boundary Layer and Sound," AlAA J., Vol. 35, No. 5, pp. 796-801, 1997.

9. Abraham, N. B., Gollub, J. P., and Swinney, H. L. "Testing Nonlinear Dynamics," Physica, Vol. 11D, pp. 252-264, 1984.

10. Casdagli, M. C., Iasemidis, L. D., Sackellares, J. C., Roper, S. N., Glimore, E. L., and Savit, R. S., "Characterizing Nonlinearity in Invasive EEG Recording From Temporal Lobe Epilepsy," Physica, Vol. D99, pp. 382-399, 1997.

11. Sugihara, G., and May, R. M., "Nonlinear Forecasting as a Way of Distinguishing Chaos From Measurement Error in Time Series," Nature, Vol. 344, pp. 734-754, 1990.

12. Abarbanel, D. I., Brown, R., and Kadtke, J. B., "Prediction of Chaotic Nonlinear System: Methods for Time Series With Broadband Fourier Spectra," Phys. Rev. A: Gen. Phys., Vol. 41, pp. 1782-1807, 1990.

13. Maestrello, L. "Chaotic Response of Panel Vibrations Forced by Turbulent Boundary Layer and Sound," AIAA J., Vol. 37, No. 3, pp. 289-295, 1999.

14. Nayfeh, A. H., and Mook, D. T., "Nonlinear Oscillation," Wiley, New York, 1979.

15. Virgin, L. N., and Dowell, E. H., "Nonlinear Aeroelasticity and Chaos," Computational Nonlinear Mechanics in Aerospace Engineering. pp. 531-54l, edited by S. N. Aturi, ALAA, Washington, 1992.

16. Bolottin, V. V., Grishko, A. A., Kounadis, A. N., Gantes, Ch., and Robert, J. B., "Influence of Initial Conditions on the Postcritical Behavior of a Nonlinear Aeroelastic System," Nonlin. Dyn., Vol. 15, pp. 63-81, 1998. 
17. Abarbancl, H. D. I., Brown, R., Sidorowich, J. J., and Tsimring. L. S., "The Analysis of Observed Chaotic Data in Physical Systems," Rev. Mod. Phys., Vol. 65, No. 4, p. 1331, 1993.

18. Geist. K., Parlitz, U., and Lauterborn, W., "Comparison of Different Methods for Computing Lyapunov Exponents," Progr. Theoret. Phys., Vol. 83, Issue 5, pp. 875-893, 1990.

19. Fenno, C. C., Bayliss, A., and Maestrello, L., "Interaction of Sound From Supersonic Jets With Nearby Structures," AIAA J., Vol. 36, No. 12, pp. 2153-2162, 1998.

20. Glass, L., and Mackey, M., "Pathological Conditions Resulting From Instabilities in Physiological Control System," Ann. New York Acad. of Sci., Vol. 316, p. 214, 1979.

21. Fuller, C. R., "Active Control of Vibration," Academic Press, London, 1996.

22. Aguirre, L., and Billings, S. A., "Validating Identified Nonlinear Models With Chaotic Dynamics," Intl. J. Bifur. Chaos in Appl. Sci. Eng., Vol. 4, No. 1, pp. 109-126, 1994.

23. Theiler, J., Eubank, S., and Farmar, J. D., "Testing for Nonlinearity in Time Series: The Method of Surrogate Data," Physica, Vol. D58, pp. 124-141, 1992.

24. Ruelle, D., "The Claude Bernard Lecture, 1989, Deterministic Chaos: The Science and Fiction," Proc. R. Soc. London, Vol. A 472, pp. 241-248, 1990.

25. Abarbanel, H. D. I., "Analysis of Observed Chaotic Data," pp. 25-112, Springer-Verlag, Berlin, 1996.

26. Grassberger, P., and Procaccia, I., "Measuring the Strangeness of Strange Attractors," Physical, Vol. 9, pp. 189-208, 1983.
27. Conte, R., and Dubois, M., "Lyapunov Exponents of Experimental System." Nonlinear Evolutions, pp. 767-780, edited by J. P. D. Leon, World Scientitic, Singapore, 1988.

28. Eckmann, J. P., and Ruelle, P., "Ergotic Theory Strange Attractor," Rev. Mod. Phys., Vol. 57. pp. 616-656, 1985 .

29. Eckmann, J. P., Kamphorst, O., Ruelle, D., and Ciliberto, S., "Lyapunov Exponents for Time Series," Phys. Rev. A: Gen. Phys., Vol. 34, No. 6. pp. $4971-4979,1986$.

30. Guckenheimer, J., and Holmes, P., "Nonlinear Oscillation in Dynamical Systems and Bifurcation of Vector Fields," Springer-Verlag, New York, 1983.

31. Braiman, Y., and Goldhirsch, I., "Taming Chaotic Dynamics With Weak Periodic Perturbations," Phys. Rev. Lett., Vol. 66, No. 20, pp. 2545-2548, 1991.

32. Chacon, R., and Bejarano, J. D., "Routes to Suppressing Chaos by Weak Periodic Perturbations," Phys. Rev. Lett., Vol. 71, No. 19, pp. 3103-3106, 1993.

33. Gao, J. Y., Narducci, L. S., Schulman, M., Squicciarini, M., and Yuan, J. M., "Route to Chaos in a Hybrid Bistable System With Delay," Phys. Rev., Vol. A 28, p. 2910, 1983.

34. Sreenivasan, K. R., "Transition of Turbulent in Fluid Flow and Low Dimensional Chaos," Frontier in Fluid Mechanics, pp. 41-67, edited by S. H. Davis and J. M. Lumley, SpringerVerlag, Berlin, 1985.

35. Sahay, A., and Sreenivasan, K. R., "The Search for a Low-Dimensional Characterization of a Local Climate System," Philos. Trans. R. Soc. London, Vol. A354, pp. 1715-1750, 1996. 

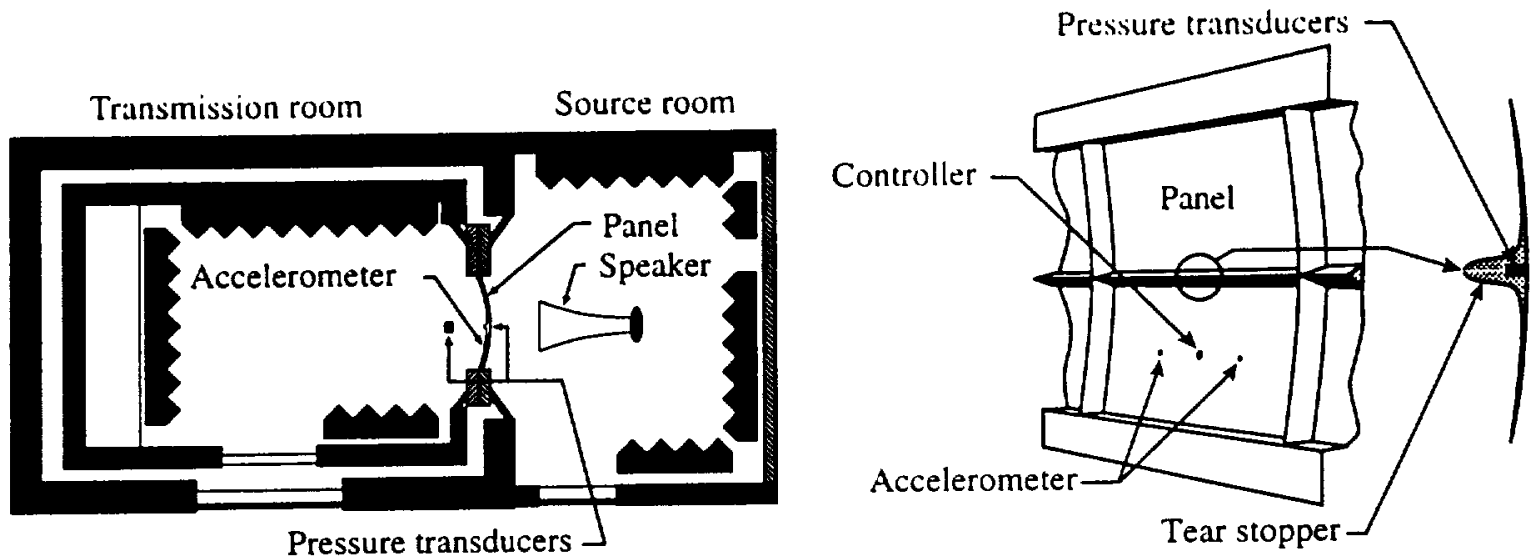

Fig. 1 Experimental setup.

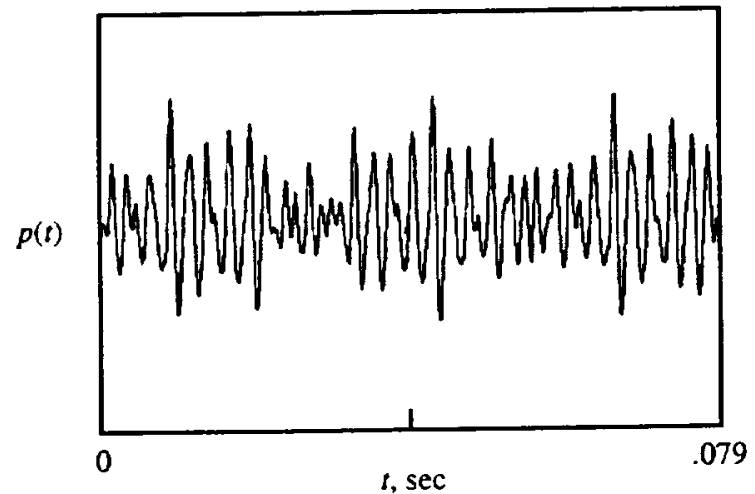

(a) Measured real-time pressure.

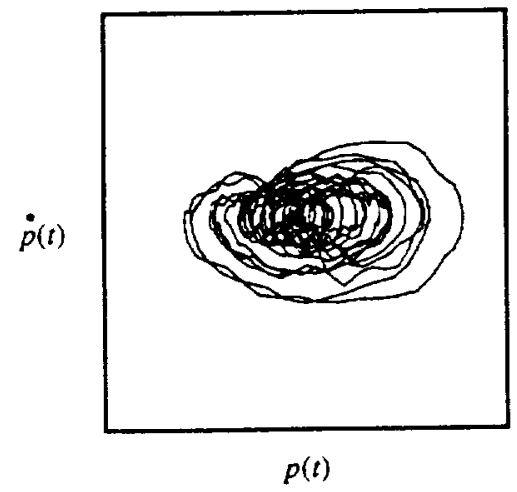

(c) Computed phase plot.

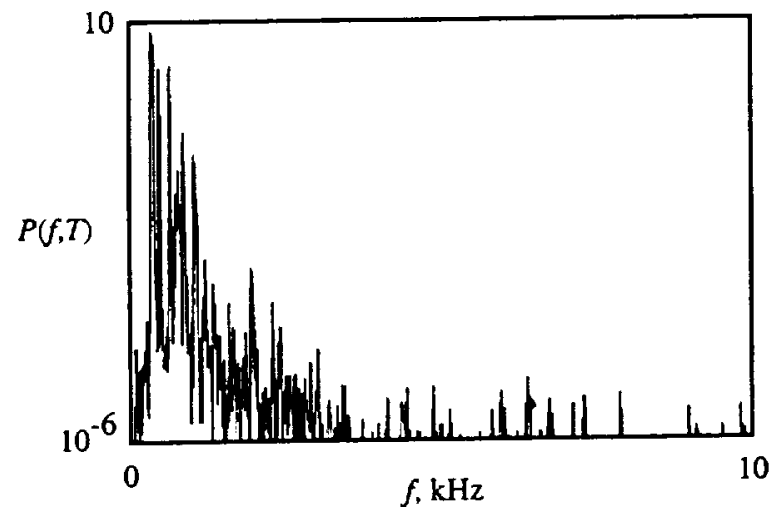

(b) Computed power spectral density.

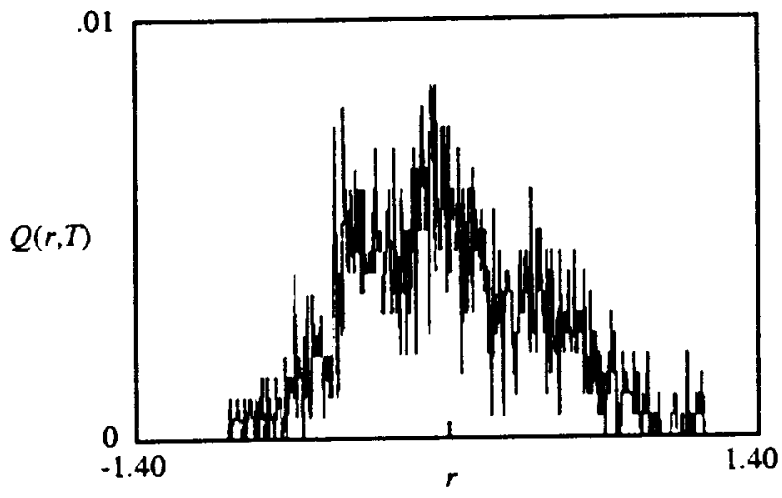

(d) Computed probability density.

Fig. 2 Normalized wall pressure fluctuation. 


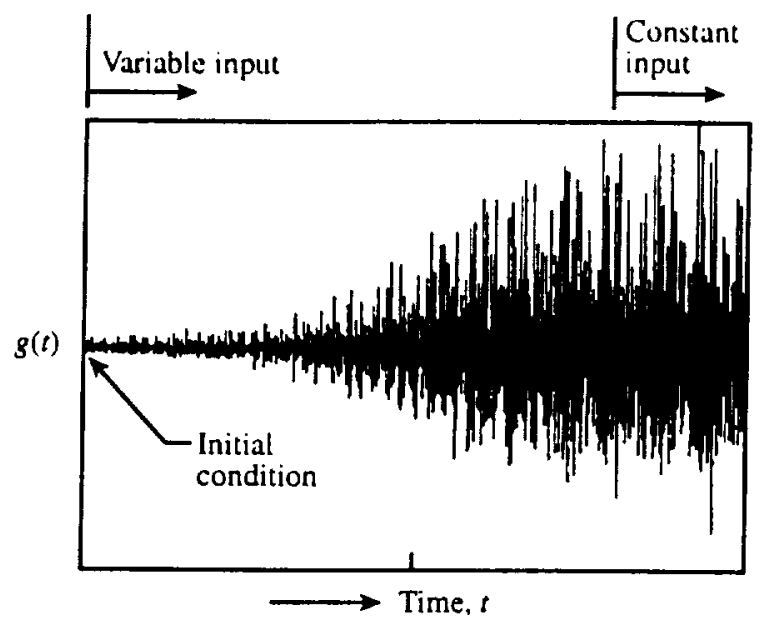

Fig. 3 Temporal evolution of the panel acceleration response.

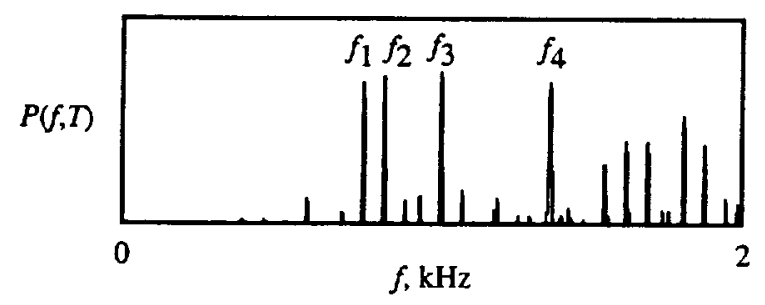

(a) Input pressure.

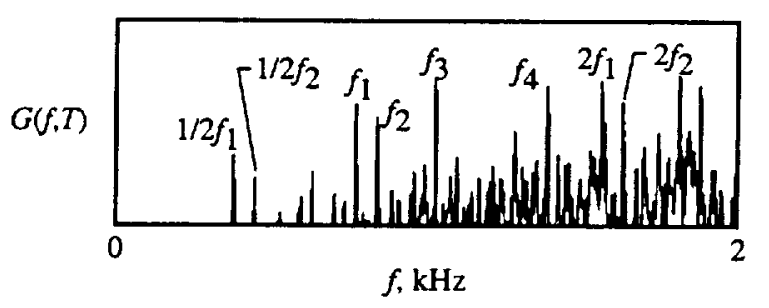

(b) Panel $r$ response.

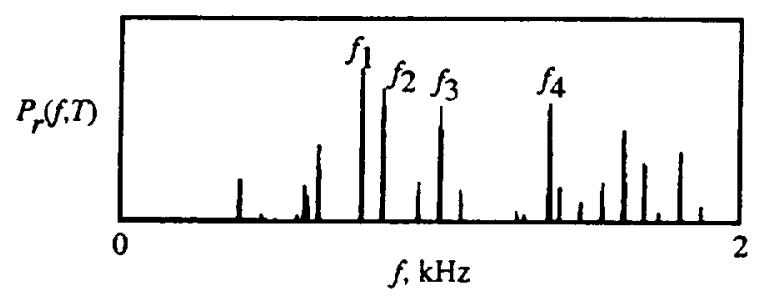

(c) Transmitted pressure.

Fig. 4 Spectral density of the initial condition. 

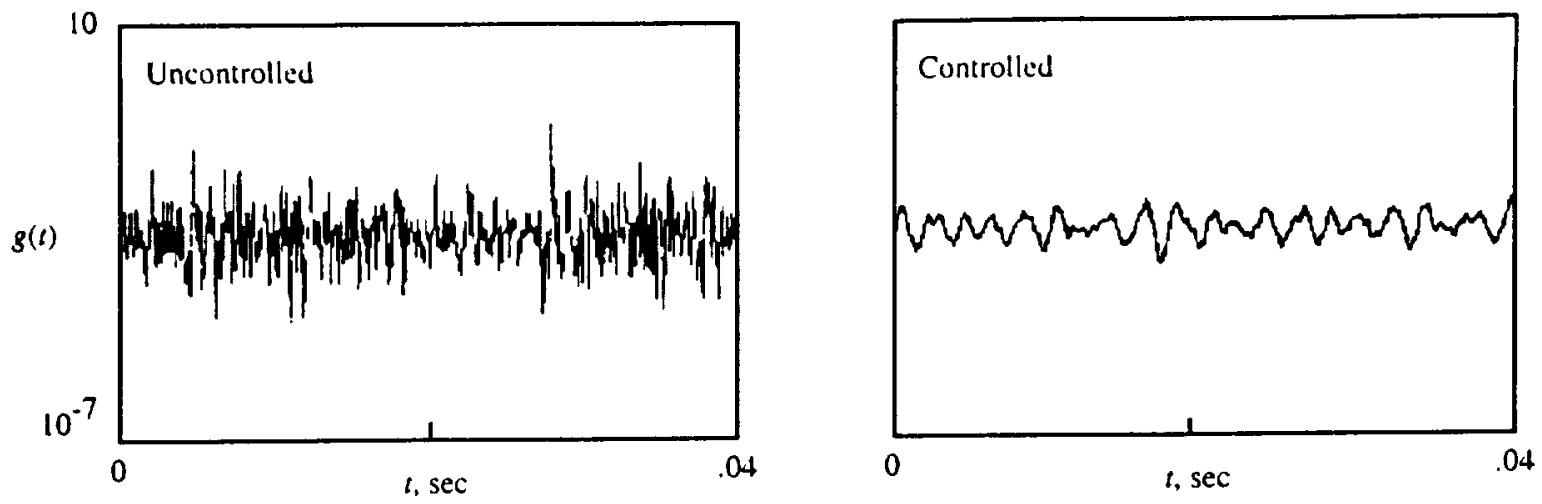

(a) Measured acceleration response.
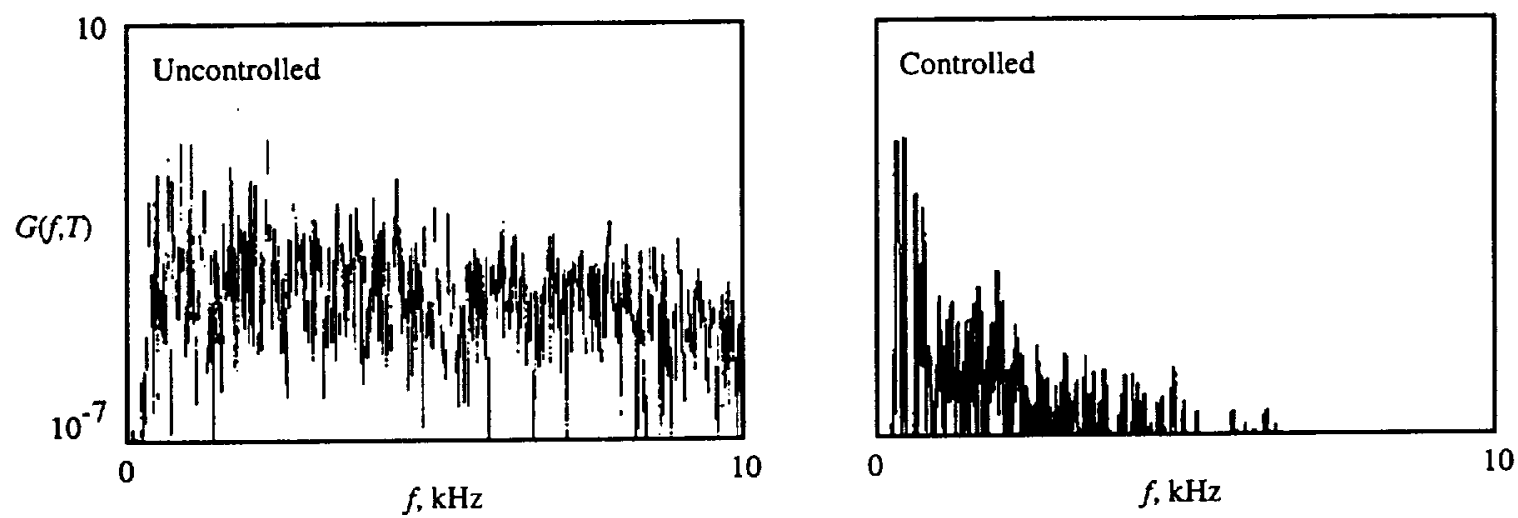

(b) Computed power spectral density.
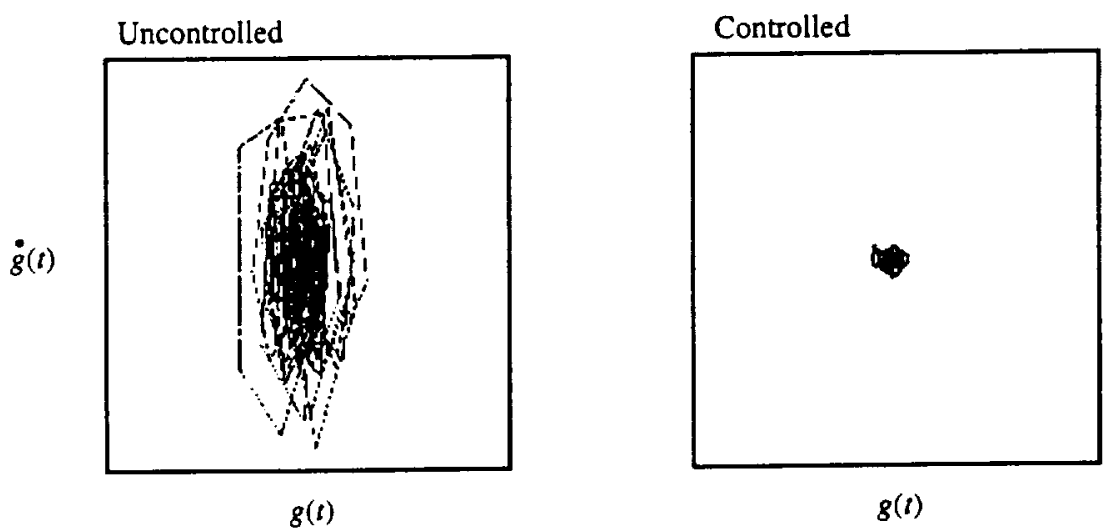

(c) Computed phase plot.

Fig. 5 Panel acceleration response at 1/4 panel length. 

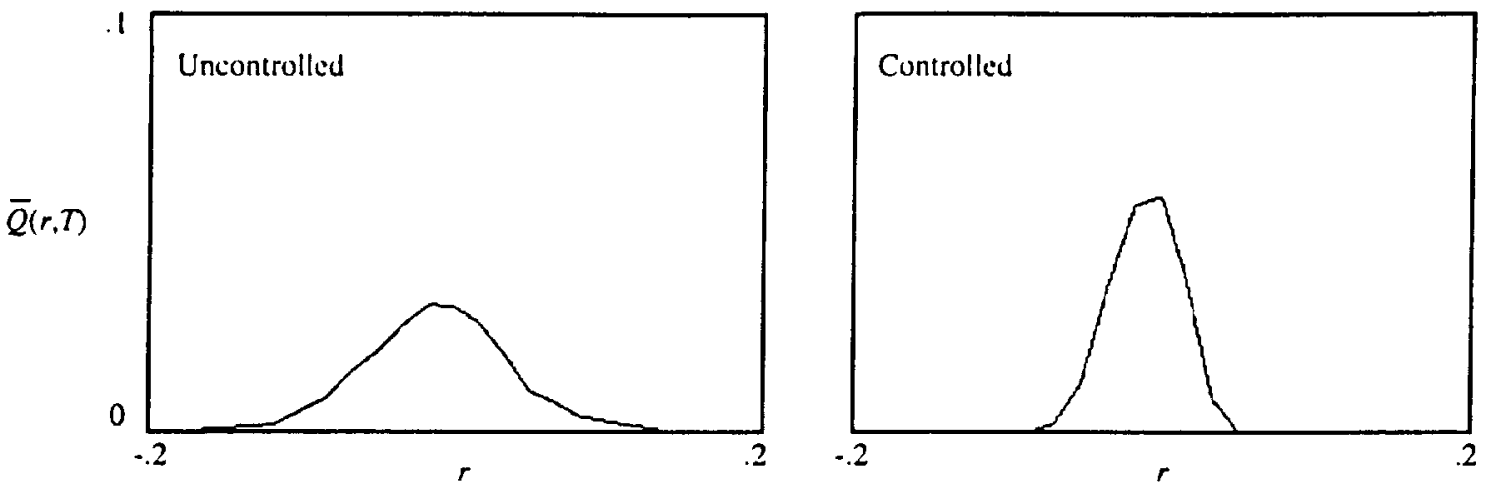

(d) Computed probability density.
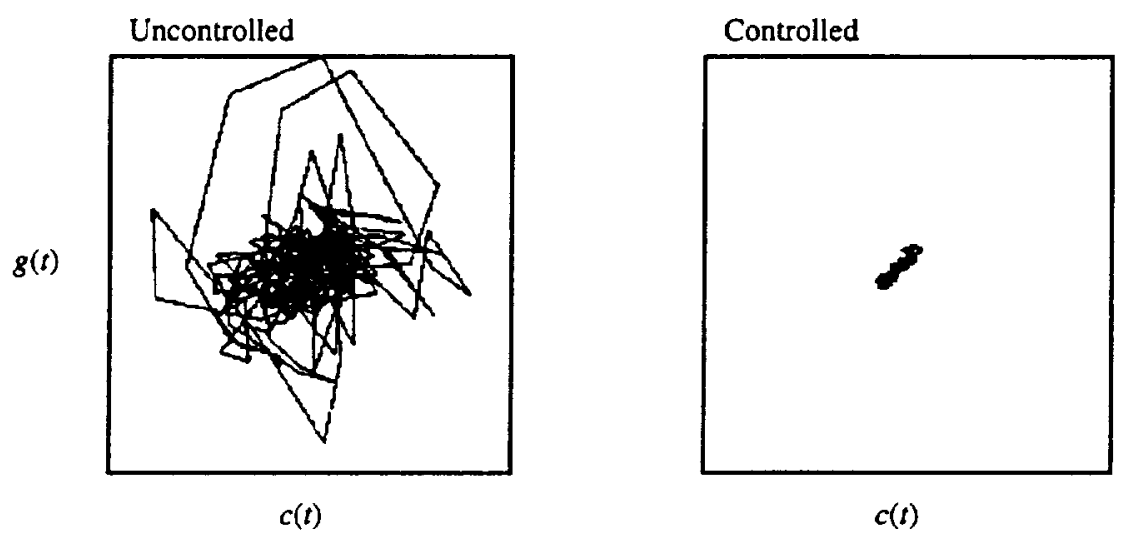

(e) Panel response versus controller response.
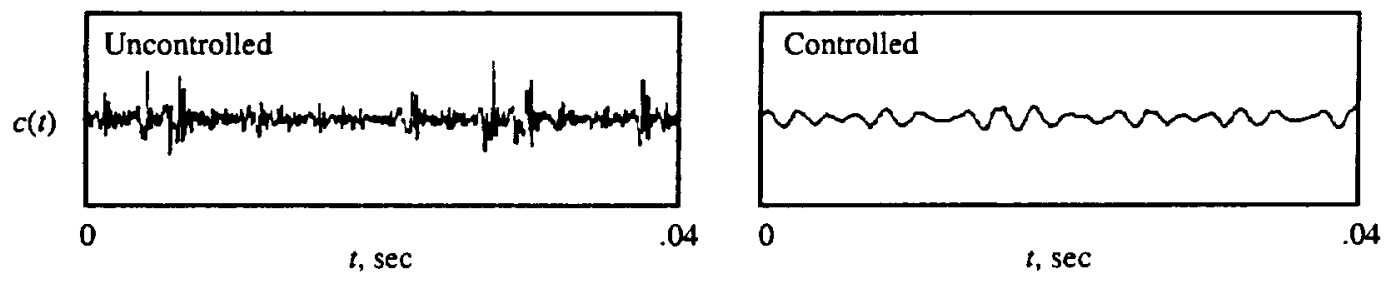

(f) Controller response.

Fig. 5 Concluded. 

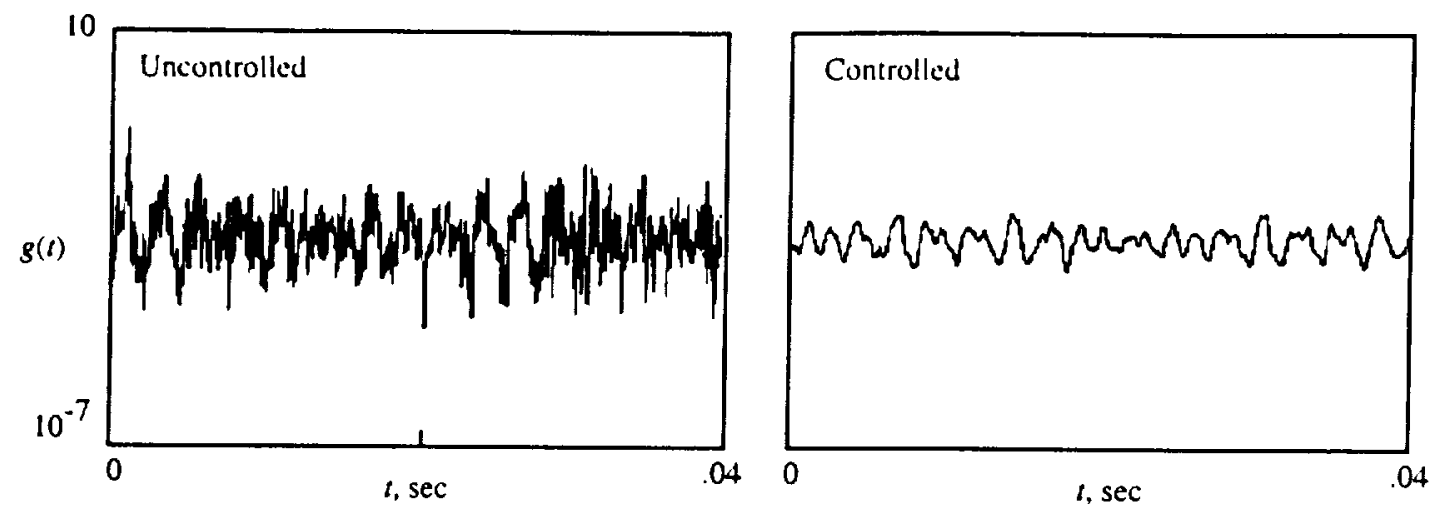

(a) Measured acceleration response.
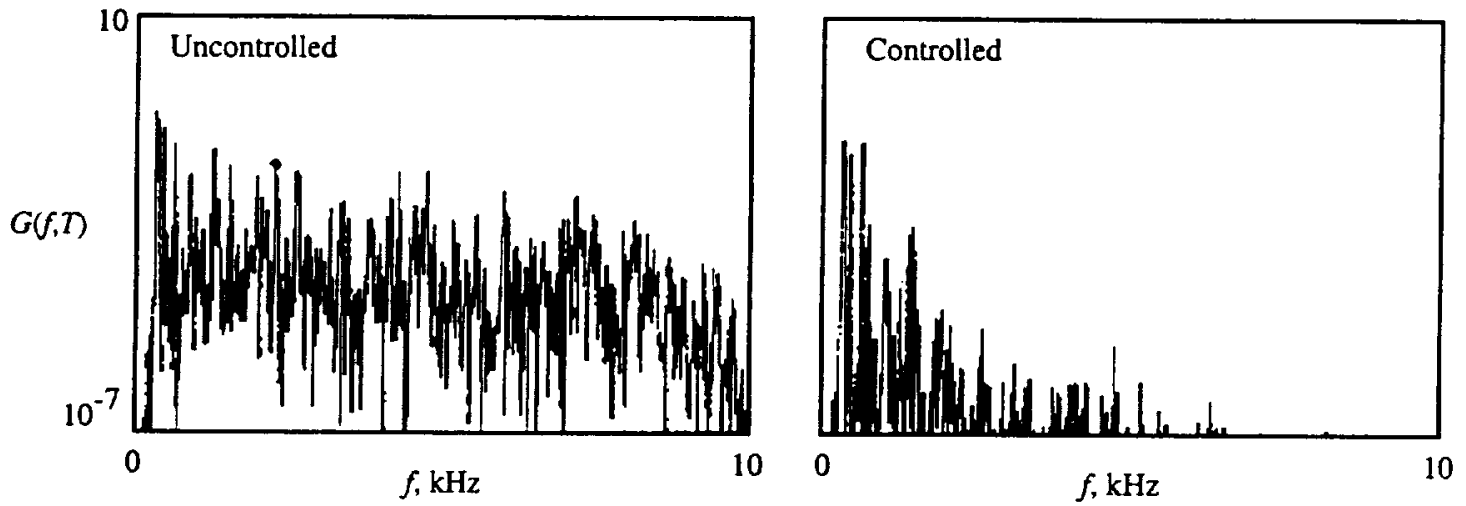

(b) Computed power spectral density. 

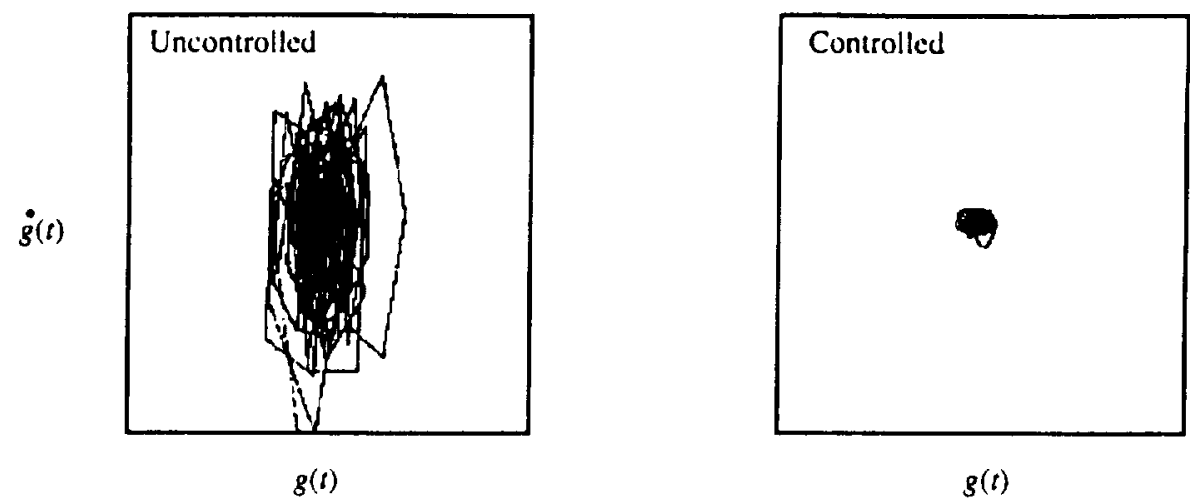

(c) Computed phase plot.

Fig. 6 Panel acceleration response at 3/4 panel length.
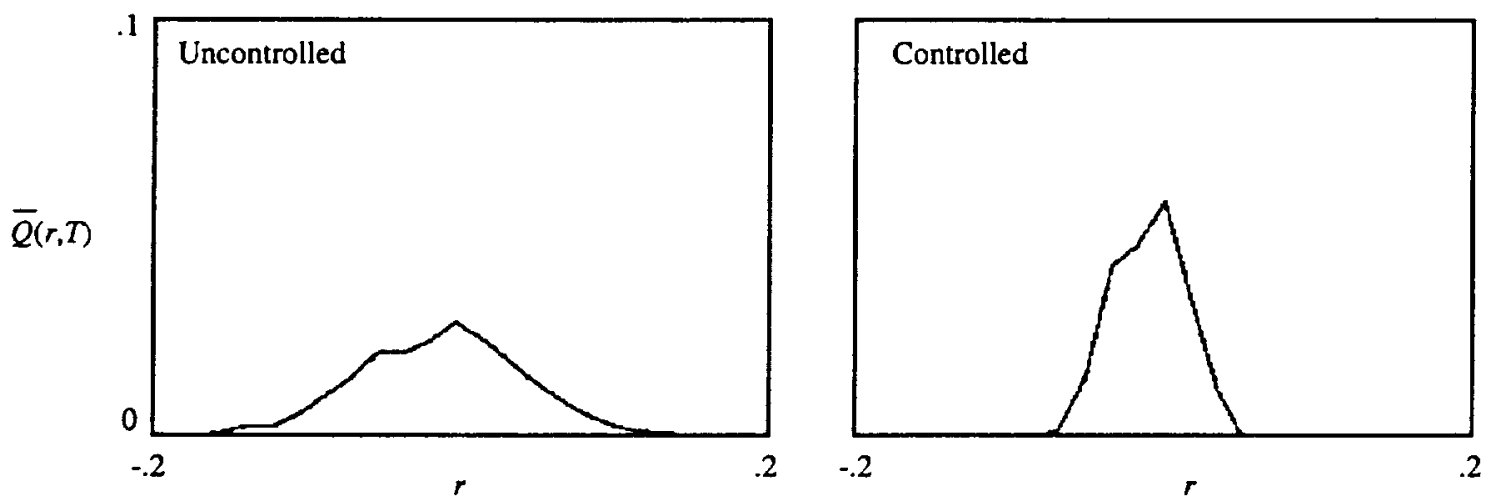

(d) Computed probability density.

Fig. 6 Concluded. 


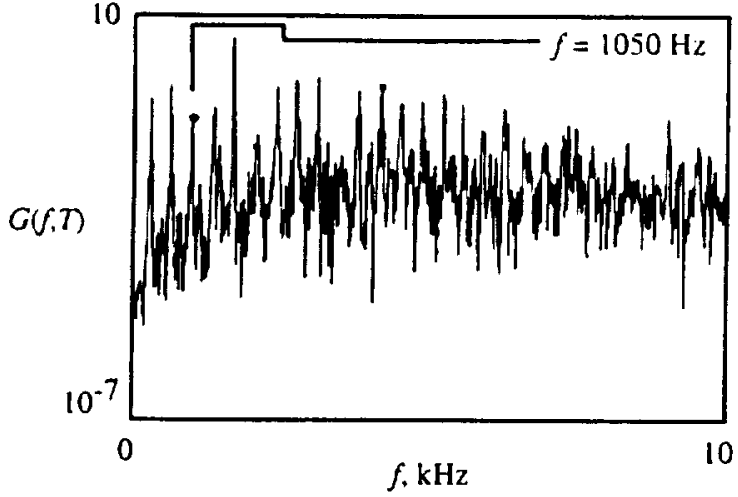

(a) Uncontrolled with initial condition.

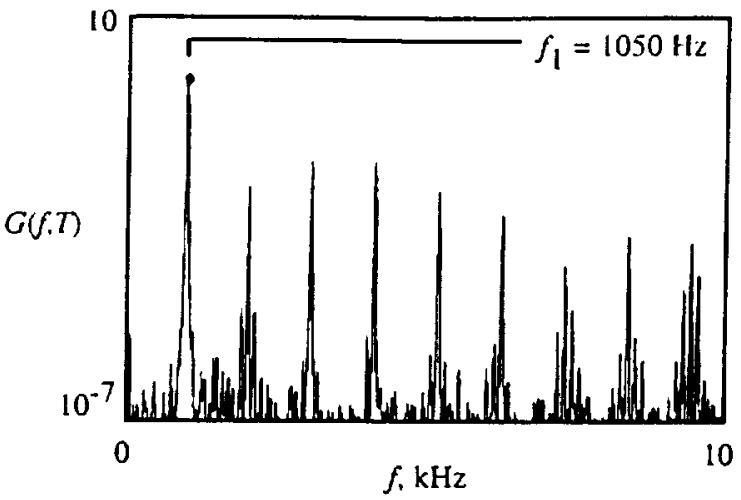

(b) Controlled with initial condition.

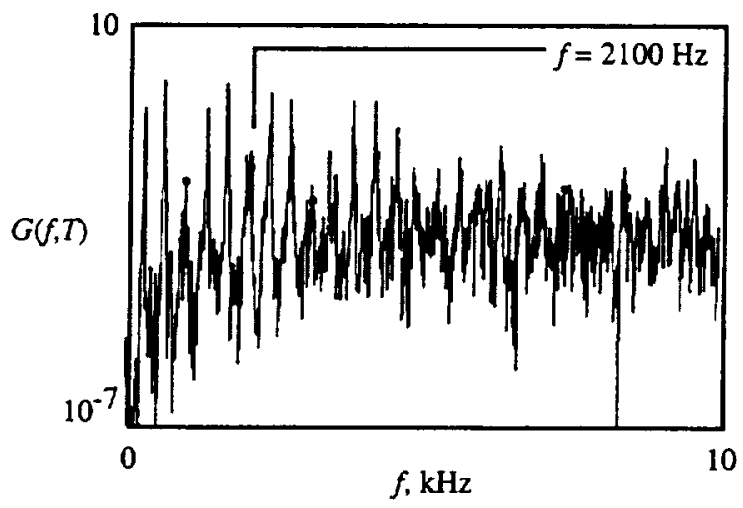

(c) Controlled with different initial condition.

Fig. 7 Panel acceleration response at $1 / 4$ panel length single frequency loading.

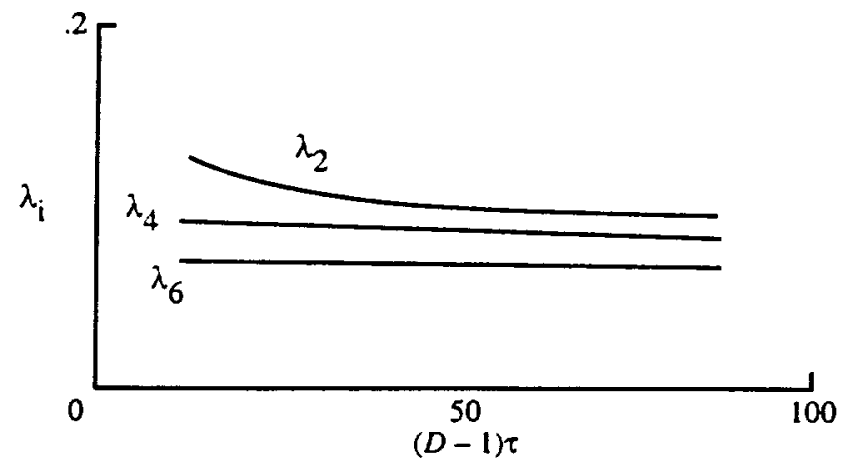

Fig. 8 Lyapunov exponent function of embedding time. 